\title{
AsialoGM1 and integrin a2ß1 mediate prostate cancer progression
}

\author{
SEVERINE VAN SLAMBROUCK ${ }^{*}$, JOHN HILKENS ${ }^{2}$, MARCO BISOFFI $^{3}$ and WIM F.A. STEELANT ${ }^{1^{*}}$ \\ ${ }^{1}$ Laboratory of Biochemical and Biomedical Research, Department of Chemistry, New Mexico Institute of Mining and \\ Technology, NM, USA; ${ }^{2}$ Department of Molecular Genetics, The Netherlands Cancer Institute, The Netherlands; \\ ${ }^{3}$ Department of Biochemistry and Molecular Biology, University of New Mexico, NM, USA
}

Received May 14, 2009; Accepted June 25, 2009

DOI: 10.3892/ijo_00000381

\begin{abstract}
The most lethal aspect of cancer is the metastatic spread of primary tumors to distant sites. Any mechanism revealed is a target for therapy. In our previous studies, we reported that the invasive activity of the bone metastatic $\mathrm{C} 4-2 \mathrm{~B}$ prostate cancer cells could be ascribed to the reorganization of the $\alpha 2 \beta 1$ integrin receptor and the $\alpha 2$ subunit-mediated association and activation of downstream signaling towards the activation of MMPs. In the present study, we demonstrate that expression of asialoGM1 in C4-2B cells correlates with cancer progression by influencing adhesion, migration and invasion, via reorganization of asialoGM1 and colocalization with integrin $\alpha 2 \beta 1$. These observations reveal an uncharacterized complex of asialoGM1 with the integrin $\alpha 2 \beta 1$ receptor promoting cancer metastatic potential through the previously identified integrin-mediated signaling pathway. The present findings promote further understanding of mechanisms by which glycosphingolipids modulate malignant properties and the results obtained here propose novel directions for future study.
\end{abstract}

\section{Introduction}

Glycosphingolipids (GSLs), including gangliosides are common components of cell membranes. They are known to

Correspondence to: Dr Severine Van Slambrouck or Dr Wim F.A. Steelant, Department of Chemistry, Laboratory of Biochemical and Biomedical Research, New Mexico Tech, 801 Leroy Place, Socorro, NM 87801, USA

E-mail: severine@nmt.edu steelant@nmt.edu

*Contributed equally

Abbreviations: ECM, extracellular matrix; FAK, focal adhesion kinase; GSL, glycosphinglipid; JNK, c-jun $\mathrm{NH}_{2}$ terminal kinase; MMP, matrix metalloproteinase

Key words: adhesion, migration, invasion, extracellular matrix, glycosphingolipid, prostate cancer serve as major cell surface antigens, in particular tumorassociated antigens, as receptors for bacterial and viral toxins, involved in the process of microbial infection, and as mediators of cell adhesion/recognition and to modulate cell motility and signal transduction (1).

Several studies indicate that the expression of tumorassociated GSLs result from oncogenic transformation, and suggest that GSL expression varies with metastatic potential $(2,3)$. Such a correlation has been shown for human melanoma and glioma cells as well as for renal cell carcinoma, where specific GSLs are found to be selectively expressed in invading tumor cells (4-6). Basically all GSLs, in tumor and normal cells, are clustered, interact with specific cell surface components and organize with intracellular signaling molecules, thereby profoundly affecting cellular function and phenotype $(1,7)$.

In our previous study, we reported that the adhesive and invasive behavior of the bone metastatic derivative $\mathrm{C} 4-2 \mathrm{~B}$ cells of the LNCaP prostate cancer progression model are mediated through the lateral reorganization of the integrin $\alpha 2 \beta 1$ receptor and the $\alpha 2$ subunit-mediated association and subsequent activation of the FAK/src/paxillin/JNK/Rac pathway, resulting in enhanced activation of matrix metalloproteinases, MMP-2 and MMP-9 (8). In addition, we pointed out that the obtained results should not be viewed as the mechanism of a single player, instead we evoke that $\alpha 2 \beta 1$ integrin receptors are key components of a complex with other membrane proteins and lipids, initiating downstream signaling events via the $\alpha 2$ subunit of the integrin $\alpha 2 \beta 1$ receptor in C4-2B cells. Since GSLs are known to play crucial roles in integrin-mediated cell adhesion, migration and invasion through their effect on signal transduction pathways, which are influenced by cross-talk with other functional membrane components including tetraspanins and growth factor receptors $(1,9,10)$, we continued our studies by investigating a possible functional role for GSLs in complex with $\alpha 2 \beta 1$ integrin receptors in the $\mathrm{LNCaP}$ progression model.

In this study, we demonstrate that in addition to the previously reported differences between $\mathrm{LNCaP}$ and $\mathrm{C} 4-2 \mathrm{~B}$ cells, namely increased adhesiveness to and invasiveness into collagen I of C4-2B cells (8), the velocity at which C4-2B cells migrate is higher as compared to the parental $\mathrm{LNCaP}$ cells. Furthermore, we demonstrate that the cell surface expression 
of asialoGM1 correlates with cancer progression and that asialoGM1 is a crucial factor to regulate cancer metastatic potential and this via lateral redistribution of asialoGM1 and colocalization with integrin $\alpha 2 \beta 1$ receptors. In conclusion, this is the first study to report that asialoGM1 is expressed on prostate cancer cells and that asialoGM1 plays a significant role in prostate cancer progression.

\section{Materials and methods}

Antibodies and other reagents. The antibodies against integrin $\alpha 2 \beta 1, \alpha 3 \beta 1, \alpha 5 \beta 1, \alpha v \beta 3$ and $\alpha v \beta 5$ receptors, GD2 and GD3 were from Millipore (Billerica, MA). The ganglioside antibodies to GM1, asialoGM1, GM2 were from EMD Biosciences (Gibbstown, NJ), while Gb3 and GM3 antibodies were purchased from Glycotech (Gaithersburg, MD). Secondary biotinylated anti-rabbit and anti-mouse, FITC-labeled antimouse, FITC- and TR-labeled anti-rabbit secondary antibodies were from Vector Laboratories (Burlingame, CA). BCA protein assay reagent kit was from Pierce (Rockford, IL). Vectastain ABC-AmP kit was obtained from Vector Laboratories.

Cell culture. The human prostate cancer $\mathrm{LNCaP}$ cells and the derivative C4-2B cell line were a kind gift from Dr M. Bisoffi (UNM, School of Medicine, NM) (11) and were grown in RPMI-medium supplemented with $5 \% \mathrm{FBS}, 100 \mathrm{IU} / \mathrm{ml}$ penicillin, $100 \mu \mathrm{g} / \mathrm{ml}$ streptomycin (Invitrogen, Carlsbad, $\mathrm{CA}$ ) at $37^{\circ} \mathrm{C}$ equilibrated with $5 \% \mathrm{CO}_{2}$ in humidified air.

Wound healing assay/scratch assay. Cells were grown in 6-well plates until confluency and washed twice with PBS. After wounding the cells, $3 \mathrm{ml}$ of medium in the presence or absence of function blocking integrin antibodies and/or ganglioside antibodies were added. After 24 and $48 \mathrm{~h}$, the distances over which the cells migrated were measured and expressed as migratory velocity $(\mu \mathrm{m} / \mathrm{h})$.

Flow cytometry analysis. Cells were detached and suspended as single cells using $10 \mathrm{mM}$ EDTA and $20 \mathrm{mM}$ HEPES buffer in the appropriate medium. The EDTA was neutralized with $\mathrm{CaCl}_{2}$ and $\mathrm{MgSO}_{4}$, and washed again with medium containing $0.1 \%$ BSA. For permeabilization, cells were incubated with Tween-20 (0.5\% in PBS). Cells $\left(2.5 \times 10^{5}\right)$ were incubated with asialoGM1 and GM1 antibodies, followed by secondary FITC-labeled antibodies. After washing, $1 \times 10^{4}$ stained cells were analyzed for fluorescence using the Cell Lab Quanta SC MPL (Beckman Coulter, Miami, FL). Stainings without primary antibody were used as controls.

Cell adhesion assay. Cells were detached with trypsin/EDTA and resuspended at $5 \times 10^{5}$ cells/ml in RPMI supplemented with $2 \%$ FBS in the presence or absence of function blocking integrin $\alpha 2 \beta 1$ and/or asialoGM1 and GM1 ganglioside antibodies. Cell suspension (100 $\mu 1)$ was added to collagen Iprecoated 96-well plates (BD Biosciences, San Jose, CA). After 90-min incubation at $37^{\circ} \mathrm{C}$, wells were washed three times with PBS. The remaining cells were solubilized with $0.2 \%$ Triton $\mathrm{X}-100$ and acid phosphatase activity was measured by addition of p-nitro-phenyl phosphate (Sigma). Absorbance values of the lysates were determined on a microplate reader at $405 \mathrm{~nm}$.
Collagen type I invasion assay. Six-well plates were filled with $1.25 \mathrm{ml}$ neutralized type I collagen $(0.09 \%$, Millipore) and incubated for $1 \mathrm{~h}$ at $37^{\circ} \mathrm{C}$ to allow gelification. For invasion into collagen type I, cells were harvested using trypsin/EDTA and seeded on top of collagen type I gels. Cultures were incubated for $24 \mathrm{~h}$ at $37^{\circ} \mathrm{C}$ in the presence or absence of function blocking integrin $\alpha 2 \beta 1$ and/or asialoGM1 and GM1 ganglioside antibodies. Numbers of cells penetrating into the gel or remaining at the surface were counted, using an inverted microscope and expressed as the invasion index, being the percentage of invading cells over the total number of cells (12).

\section{Confocal-fluorescence immunostaining}

Fluorescence microscopy. Cells were suspended in trypsin/ EDTA and stained by an indirect immunofluorescence technique. Briefly, cells were incubated on a rotator at $4^{\circ} \mathrm{C}$ for $1 \mathrm{~h}$ with antibodies against integrin $\alpha 2 \beta 1$, asialoGM1 and GM1, washed three times with CMF-HBSS and fixed with $3 \%$ paraformaldehyde in CMF-HBSS for 20 min. Fixed cells were washed three times with CMF-HBSS and incubated with FITC-labeled anti-mouse secondary antibody. Stained cells were mounted with a drop of Glycergel mounting medium (Dako Corp., Carpinteria, CA) containing 1\% 1,4-diazabicyclo[2.2.2] octane (fluorescence stabilizer). Fluorescence was observed by fluorescence microscopy (Olympus IX51 with Olympus U-CMAD3 camera).

Confocal microscopy. Cells were grown on glass cover slips (diameter, $12 \mathrm{~mm}$ ) placed in 24-well plates. The glass cover slips were removed, washed and fixed with $3 \%$ paraformaldehyde. Next, fixed cells were washed and incubated with primary antibodies against asialoGM1, GM1 and integrin $\alpha 2 \beta 1$, followed by incubation with FITC-labeled and TRlabeled secondary antibodies. Stained cells were mounted with Glycergel mounting medium (Dako) containing 1\% 1,4diazabicyclo[2.2.2] octane (fluorescence stabilizer) and confocal fluorescence images were acquired using a Leica TCS SP2 confocal system (Leica Microsystems, Heidelberg, Germany). Images were taken using a 63x NA 1.32 objective. The standard LCS software was used. Cross-talk between different fluorochromes, which could give rise to false-positive colocalization of the signals, was totally avoided by careful selection of the imaging conditions. Standard filter combinations and Kalman averaging was used.

Co-immunoprecipitation of cell surface molecules. Cells at $70 \%$ confluency were lysed, using $0.5 \mathrm{ml}$ lysis buffer containing $1 \%$ Triton X-100, 1\% NP-40 and the following inhibitors: aprotinin $(10 \mu \mathrm{g} / \mathrm{ml})$, leupeptin $(10 \mu \mathrm{g} / \mathrm{ml})$, PMSF $(1.72 \mathrm{mM}), \mathrm{NaF}(100 \mathrm{mM}), \mathrm{NaVO}_{3}(500 \mu \mathrm{M})$, and $\mathrm{Na}_{4} \mathrm{P}_{2} \mathrm{O}_{7}$ $(500 \mu \mathrm{g} / \mathrm{ml})$. Lysates, containing $2000 \mu \mathrm{g}$ protein, were mixed with protein G-Sepharose beads (Amersham Biosciences, $\mathrm{NJ})$ to preclear non-specific binding. Antibody to asialoGM1 $(1: 100)$ was added to the collected supernatant and rotated at $4^{\circ} \mathrm{C}$ overnight. Subsequently, protein G-Sepharose beads were used to recover the immunocomplexes. Immunoprecipitates were resolved in $150 \mu 1$ SDS-PAGE sample buffer and heated to $95^{\circ} \mathrm{C}$ for $5 \mathrm{~min}$. The supernatants were electrophoresed on $7.5 \%$ SDS-PAGE and transferred to PVDF membranes (Immobilon-P) (Bio-Rad Laboratories, Hercules, CA). After 


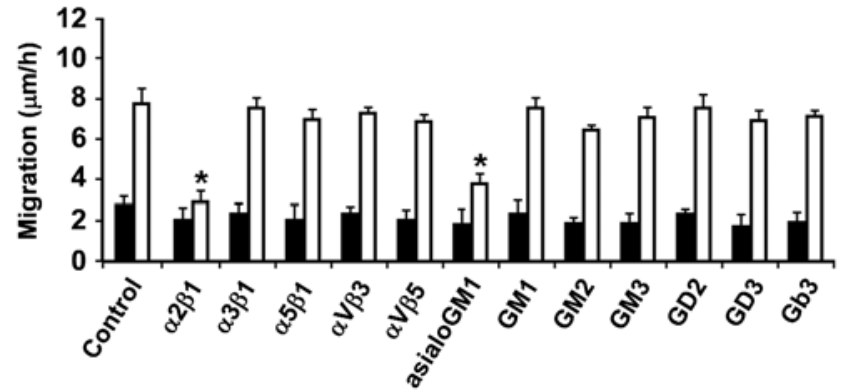

Figure 1. Migratory differences between LNCaP and C4-2B cells. Migration of LNCaP (closed bars) and C4-2B (open bars) cells. Cells grown in 6-well plates until confluency, were wounded and allowed to grow in the absence or presence of function blocking integrin $(5 \mu \mathrm{g} / \mathrm{ml})$ and/or ganglioside (1:500) antibodies. After 24 and $48 \mathrm{~h}$, the distances over which the cells migrated were measured and results are expressed as migratory velocity $(\mu \mathrm{m} / \mathrm{h})$. Asterisks indicate statistical difference from untreated C4-2B cells $(\mathrm{p}<0.05)$.

transfer, membranes were incubated with antibody against integrin $\alpha 2 \beta 1$, followed by incubation with a secondary biotinylated antibody and developed by ECL (Vectastain $\mathrm{ABC}-\mathrm{AmP}$ ) detection kit. Membranes were imaged on the BioChemi System and analysis software (UVP, Upland, CA).

Statistical analyses. All treatments were matched and carried out at least three times. Data were analyzed using Excel, for determination of mean, SD, and Student's t-test (95\%). Intensity of the immunoblotted bands was quantified by densitometry, using statistical software Scion Image (Scion Corp., Frederick, MD).

\section{Results}

Migratory differences between LNCaP and C4-2B cells. In addition to the previously reported differences between LNCaP and C4-2B cells, namely increased adhesiveness to and invasiveness into collagen I of $\mathrm{C} 4-2 \mathrm{~B}$ cells (8), we report herein that the velocity at which $\mathrm{C} 4-2 \mathrm{~B}$ cells migrate is higher as compared to the parental LNCaP cells (Fig. 1). Treatment with function blocking integrin and ganglioside antibodies reveals that integrin $\alpha 2 \beta 1$ antibody is the sole integrin antibody able to inhibit the migratory capacity of C4-2B cells, while asialoGM1 is the major ganglioside involved in the migration of $\mathrm{C} 4-2 \mathrm{~B}$ cells as compared to the parental LNCaP cells (Fig. 1).

Cell surface and total expression of asialoGM1. We reported that there are no significant differences in the total expression and cell surface expression levels of integrin $\alpha 2 \beta 1$ in LNCaP and C4-2B cells (8). Next, we examined the cell surface (left panel) and total expression (right panel) levels of asialoGM1 on LNCaP and C4-2B cells by flow cytometry with respective antibodies, given the minor difference between asialoGM1 and GM1 (Fig. 2). The left panel of Fig. 2 shows that

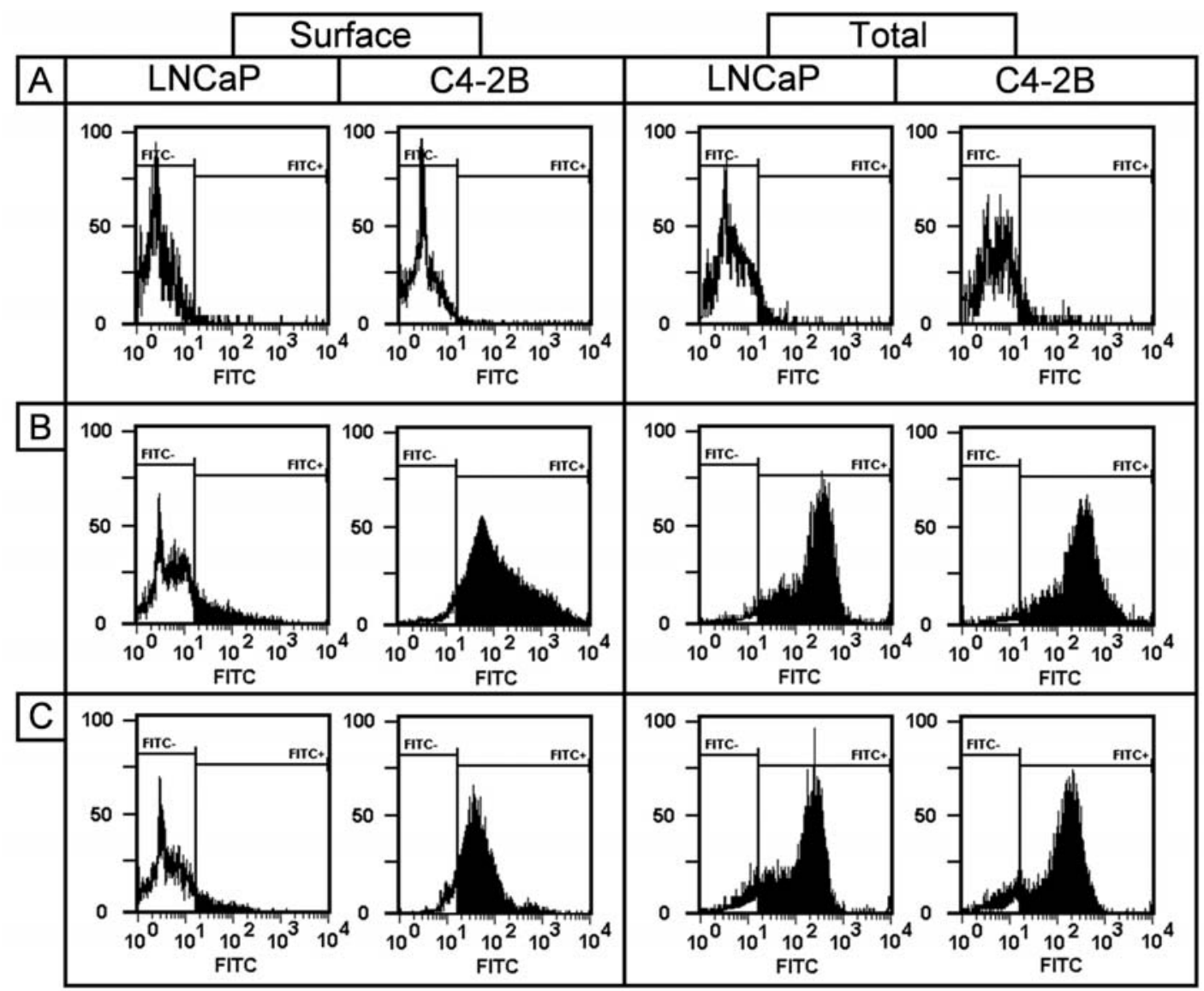

Figure 2. Cell surface and total expression levels of asialoGM1 and GM1. Left panel, cell surface expression levels of asialoGM1 (B) and GM1 (C) in LNCaP and C4-2B cells. Single cell suspensions were stained by relevant primary antibodies and FITC-labeled secondary antibodies. Right panel, total expression levels of asialoGM1 (B) and GM1 (C) in LNCaP and C4-2B cells, after permeabilization with Tween-20 (0.5\% in PBS), followed by incubation with primary antibodies and FITC-labeled secondary antibodies. Control stainings were performed without primary antibody (A). Experiments were performed at least three times. 


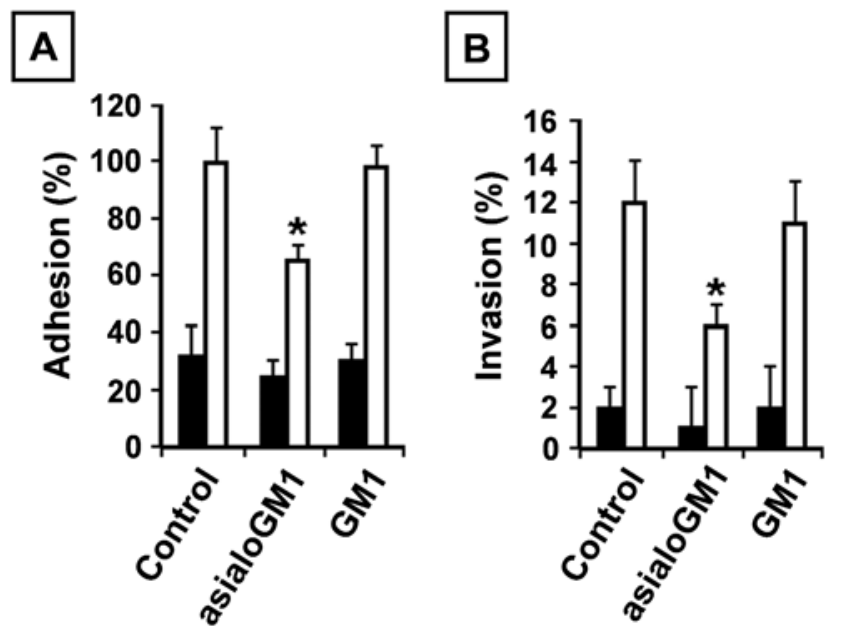

Figure 3. AsialoGM1 mediates adhesion and invasion. (A) Adhesion to collagen I. LNCaP $\left(5 \times 10^{4}\right)$ (closed bars) and C4-2B (open bars) cells/100 $\mu 1$, untreated or treated with asialoGM1 or GM1 antibodies (1:500) were seeded in a collagen I precoated 96-well plate. After $90 \mathrm{~min}$, the cells were washed and acid phosphatase activity was measured. The percentage binding was calculated by subtracting non-specific binding and expressed as \% adhesion compared to untreated LNCaP and C4-2B cells. Six wells were analyzed in each experiment. (B) Invasion into collagen I. LNCaP $\left(1 \times 10^{4}\right)$ (closed bars) and C4-2B (open bars) cells, untreated or treated with asialoGM1 or GM1 antibodies (1:500), were seeded on top of collagen type I gels and cultured for $24 \mathrm{~h}$. The invasion index expresses the percentage of invading cells over the total number of cells. All data are means \pm SD from three independent experiments, asterisks indicate statistical difference from untreated $\mathrm{C} 4-2 \mathrm{~B}$ cells $(\mathrm{p}<0.05)$.

asialoGM1 (Fig. 2B) and GM1 (Fig. 2C) are expressed at the cell surface of $\mathrm{C} 4-2 \mathrm{~B}$ cells and could not be detected in the parental LNCaP cells as compared to the respective controls (Fig. 2A). The total expression levels were determined after permeabilization and the results in Fig. 2 (right panel) reveal that total expression levels of asialoGM1 (Fig. 2B) and GM1 (Fig. 2C) are comparable for both cell lines. In summary, the data suggest that the increased cell surface expression levels of asialoGM1 and GM1 in the bone metastatic derivative cell line might correlate with cancer progression.

AsialoGM1 mediates adhesion and invasion. Given the pronounced cell surface expression of both asialoGM1 and GM1 in $\mathrm{C} 4-2 \mathrm{~B}$ cells and the profound influence of asialoGM1 on the migration of $\mathrm{C} 4-2 \mathrm{~B}$ cells, we investigated whether asialoGM1 and/or GM1 were involved in adhesion to and invasion into collagen $\mathrm{I}$. The results reveal that treatment with GM1 antibody does not affect any of these processes, while asialoGM1 antibody treatment significantly reduces the adhesion to collagen I (Fig. 3A), as well as the invasiveness into collagen I of C4-2B cells (Fig. 3B). Overall, these results suggest that asialoGM1 plays a role in the different processes involved in cancer progression, i.e. adhesion, migration (Fig. 1) and invasion.

Organization of asialoGM1 and colocalization with integrin $\alpha 2 \beta 1$. The integrin $\alpha 2 \beta 1$ receptors were found reorganized in the derivative bone metastatic $\mathrm{C} 4-2 \mathrm{~B}$ cells and these changes in organizational pattern correlated with increased adhesion and cellular invasion. The major alterations in the organizational status, rather than increased expression, were responsible

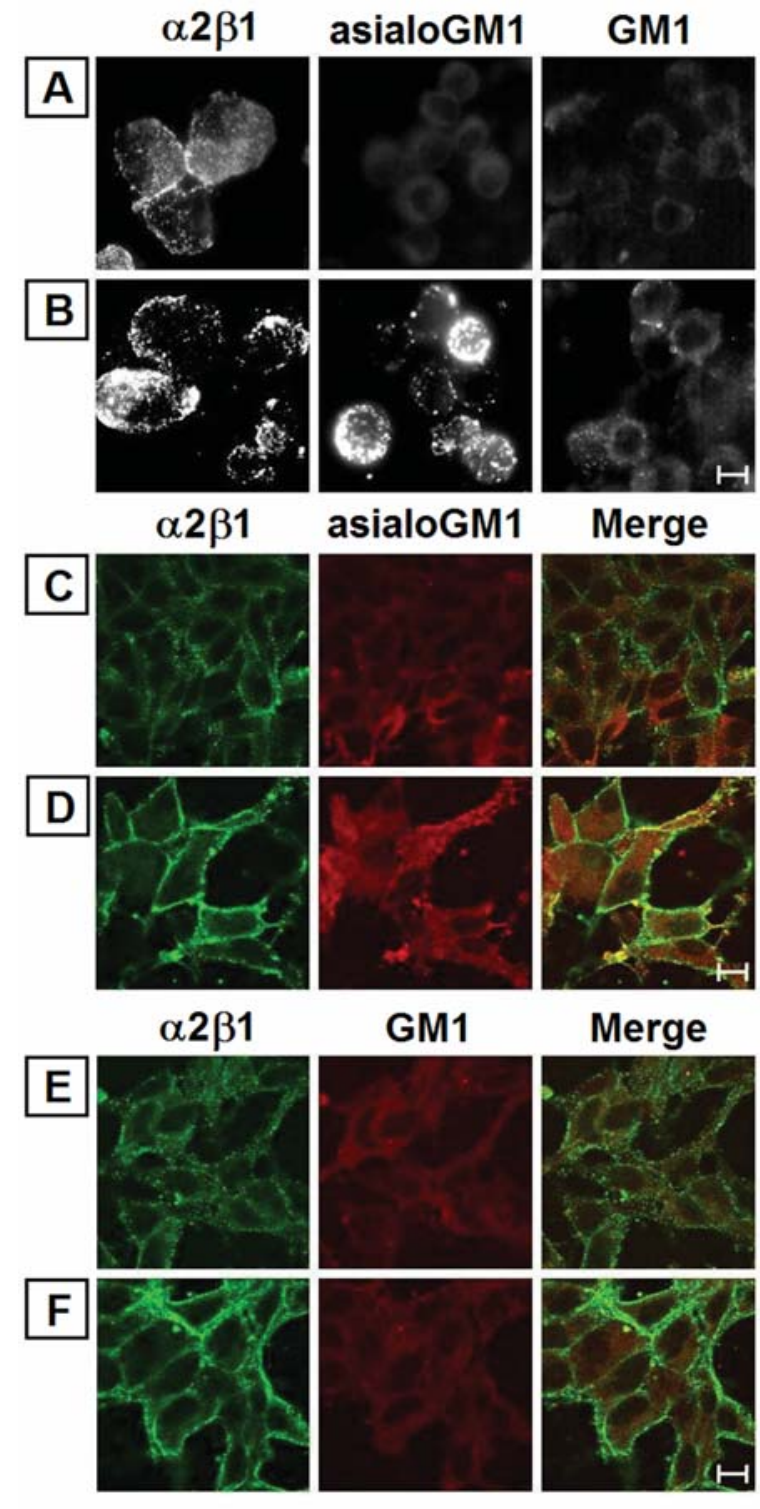

G

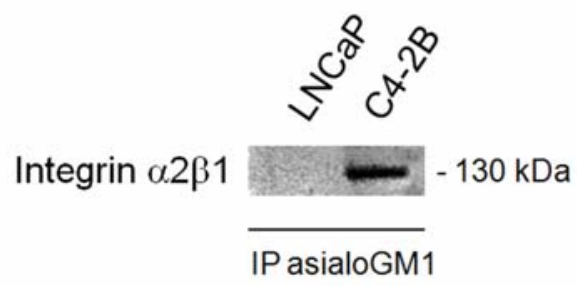

Figure 4. Organization of asialoGM1 and colocalization with integrin $\alpha 2 \beta 1$. Organization of integrin $\alpha 2 \beta 1$, asialoGM1 and GM1 by indirect fluorescence (A and B) and confocal microscopy (C-F). (A and B) Cell surface staining pattern of integrin $\alpha 2 \beta 1$, asialoGM1 and GM1 in LNCaP (A) and C4-2B (B) cells as analyzed by fluorescence microscopy. Cells were suspended in trypsin/EDTA and incubated with relevant primary antibodies on a rotator at $4^{\circ} \mathrm{C}$ for $1 \mathrm{~h}$, fixed with $3 \%$ paraformaldehyde in CMF-HBSS and labeled with secondary FITC-labeled antibody. Reorganized staining patterns for integrin $\alpha 2 \beta 1$ and asialoGM1 are observed in C4-2B cells. (C-F) Colocalization of integrin $\alpha 2 \beta 1$ and asialoGM1 in LNCaP and C4-2B cells studied by confocal microscopy. Cells were grown on glass cover slips and double stained by integrin $\alpha 2 \beta 1$ and asialoGM1 primary antibodies and FITC- and TR-labeled secondary antibodies. In C4-2B, integrin $\alpha 2 \beta 1$ colocalized with asialoGM1 more extensively than in LNCaP cells. Control staining, for (A-F), were performed without primary antibody (data not shown); scale bar, $10 \mu \mathrm{m}$. Experiments were performed at least three times. (G) Different level of integrin $\alpha 2 \beta 1$ /asialoGM1-association in LNCaP versus C4-2B cells. Aliqouts of immunoprecipitates were analyzed by Western blotting using the respective antibody. Experiments were performed at least three times. 

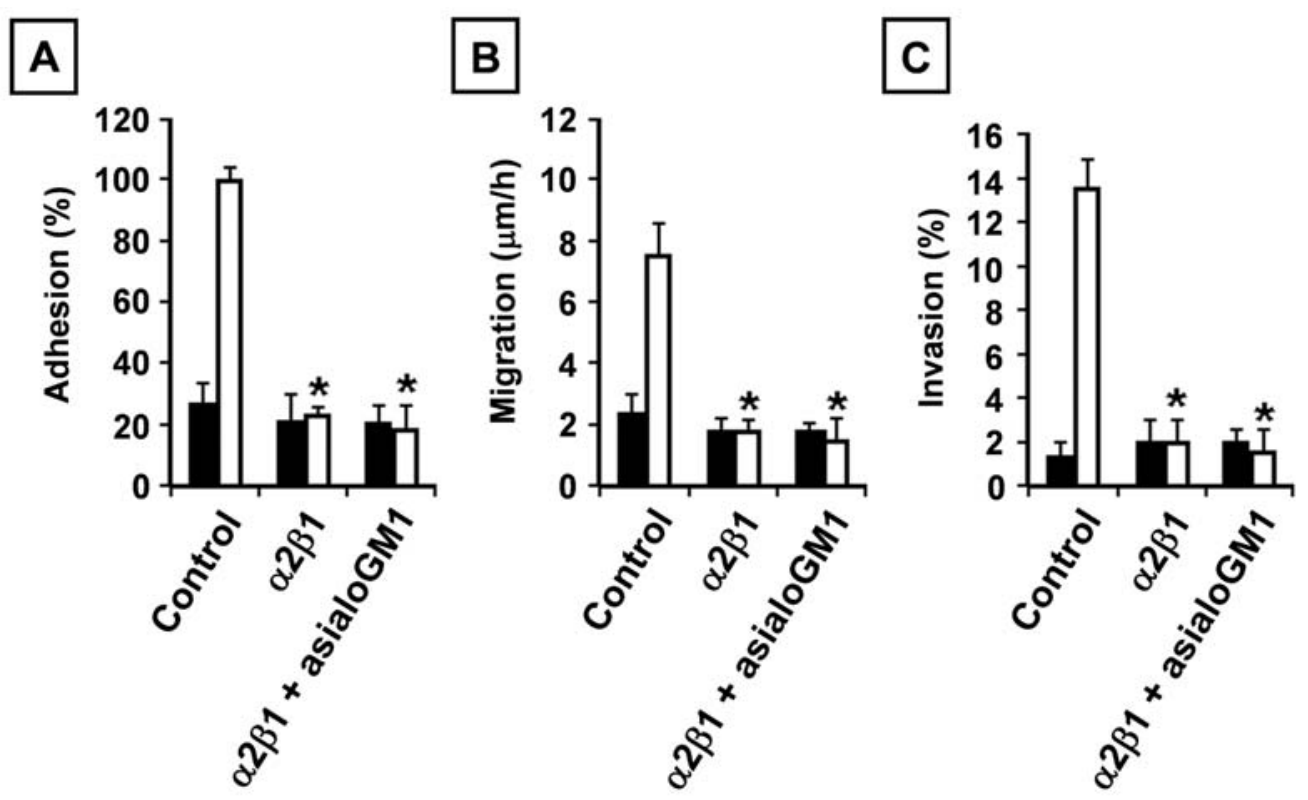

Figure 5. Is there a synergistic interaction of asialoGM1 and integrin $\alpha 2 \beta 1$ ? (A) Adhesion to collagen I. LNCaP (5x104) (closed bars) and C4-2B (open bars) cells $/ 100 \mu 1$, untreated or treated with asialoGM1 (1:500) and function blocking integrin $\alpha 2 \beta 1$ (5 $\mu$ g/ml) antibodies, were seeded in a collagen I precoated 96-well plate. After $90 \mathrm{~min}$, the cells were washed and acid phosphatase activity was measured. The percentage binding was calculated by subtracting nonspecific binding expressed as \% adhesion compared to untreated LNCaP and C4-2B cells. Six wells were analyzed in each experiment. (B) Migration. LNCaP (closed bars) and C4-2B (open bars) cells grown in 6-well plates until confluency were wounded and allowed to grow in the absence or presence of asialoGM1 (1:500) and function blocking integrin $\alpha 2 \beta 1(5 \mu \mathrm{g} / \mathrm{ml})$ antibodies. The distances over which the cells migrated were measured, after 24 and $48 \mathrm{~h}$, and results are expressed as migratory velocity $(\mu \mathrm{m} / \mathrm{h})$. (C) Invasion into collagen I. LNCaP (1x10 $)$ (closed bars) and C4-2B (open bars) cells, untreated or treated with asialoGM1 (1:500) and function blocking integrin $\alpha 2 \beta 1(5 \mu \mathrm{g} / \mathrm{ml})$ antibodies, were seeded on top of collagen type I gels and cultured for $24 \mathrm{~h}$. The invasion index expresses the percentage of invading cells over the total number of cells. All data are means \pm SD from three independent experiments, asterisks indicate statistical difference from untreated C4-2B cells $(\mathrm{p}<0.05)$.

for the initiation and activation of signal transducers in the downstream pathway, leading to invasion via association with the integrin $\alpha 2$ subunit (8). Next, we examined the localization and distribution patterns of asialoGM1 and possible colocalization with integrin $\alpha 2 \beta 1$ receptors by fluorescence and confocal microscopy. The fluorescence immunostaining of $\mathrm{LNCaP}$ and $\mathrm{C} 4-2 \mathrm{~B}$ cells in suspension reveals major alterations in cell surface staining pattern for asialoGM1 and integrin $\alpha 2 B 1$ (8) but not for GM1 in C4-2B cells (Fig. 4B), as compared to the parental LNCaP cells (Fig. 4A). The confocal microscopy images (Fig. 4C-F) also display that asialoGM1 and integrin $\alpha 2 \beta 1$ are differentially organized and clustered in C4-2B cells (Fig. 4D), as compared to the parental LNCaP cells (Fig. 4C), and asialoGM1 is found to have colocalized with integrin $\alpha 2 \beta 1$ in $\mathrm{C} 4-2 \mathrm{~B}$ cells, while no such clustering and colocalization of GM1 with integrin $\alpha 2 \beta 1$ is found in $\mathrm{LNCaP}$ (Fig. 4E) and in C4-2B (Fig. 4F) cells. The close connection is further confirmed by co-immunoprecipitation of asialoGM1, demonstrating the association of integrin $\alpha 2 \beta 1$ in $\mathrm{C} 4-2 \mathrm{~B}$ cells and not in LNCaP cells (Fig. 4G).

Possible synergistic interaction of asialoGM1 and integrin $\alpha 2 \beta 1$. Based on the above data, we wanted to determine the significance of the asialoGM1/integrin $\alpha 2 \beta 1$ interaction. Fig. 5A and $\mathrm{C}$ show that function blocking of the integrin $\alpha 2 \beta 1$ receptor blocks the adhesive and invasive behavior of C4-2B cells in collagen I, which is in line with our previously published results. Additionally, function blocking of integrin $\alpha 2 \beta 1$ reduces the migratory capacity of C4-2B cells (Fig. 1). Subsequently, we investigated whether blocking of both integrin $\alpha 2 \beta 1$ and asialoGM1 resulted in a synergistic effect. Co-treatment with antibodies against integrin $\alpha 2 \beta 1$ and asialoGM1 reduced even more the observed effects, however, not significantly different from the effect of the integrin $\alpha 2 \beta 1$ antibody by itself (Fig. 5).

\section{Discussion}

Understanding the metastatic and invasive properties of tumor cells are of crucial importance in tumor malignancy. Every possible mechanism revealed could be a target for prevention or inhibition of tumor progression. Various studies indicate that tumor cell penetration into extracellular matrix (ECM), based on increased motility coupled with ECM-destructive activity is often associated with high metastasis (13). In our previous study, we showed that the bone-metastatic derivative C4-2B cells of the LNCaP progression model display enhanced invasiveness into and adhesiveness to collagen I as compared to the parental LNCaP cells. The observed differences were mediated through the reorganization of the $\alpha 2 \beta 1$ receptor, resulting in the activation of a downstream signaling pathway leading to increased activity of matrix metalloproteinases (8).

In the present study we found that, in addition to the markedly enhanced adhesion and invasion activity, C4-2B cells migrate faster than the parental LNCaP cells. Moreover, integrin $\alpha 2 \beta 1$ and asialoGM1 were identified as important mediators of cell migration. Suppression of these two 
molecules using asialoGM1 and integrin $\alpha 2 \beta 1$ receptor antibodies clearly demonstrated their particular roles in the increased cell migration of $\mathrm{C} 4-2 \mathrm{~B}$ cells. In contrast, $\mathrm{LNCaP}$ cells were scarcely affected by these treatments. Analysis of the cell surface and total expression levels of asialoGM1 and the related GM1 revealed enhanced expression of asialoGM1, at the cell surface in C4-2B cells as compared to $\mathrm{LNCaP}$ cells. On the other hand, no significant differences in total expression and cell surface levels were found for the integrin $\alpha 2 \beta 1$ receptor in the preceding study (8).

The neutral glycosphingolipid, asialoGM1, is expressed by $\mathrm{T}$ cells as well as by natural killer (NK) cells (14) and its expression correlates with the killing activity. Traces of asialoGM1 have also been found in nerve tissues and in cystic fibrosis respiratory epithelial cells $(15,16)$. Furthermore, asialoGM1 has been described as an adhesion receptor for $P$. aeruginosa to regenerate respiratory epithelial cells $(17,18)$. Moreover, it has been reported that asialoGM1 expression could vary according to the cellular phenotype (19) which is in agreement with our observations showing that the cell surface expression of asialoGM1 correlates with cancer progression and that asialoGM1 is a crucial factor to regulate cancer metastatic potential by influencing adhesion, migration and invasion.

In the immunostaining studies, differences in organizational pattern of asialoGM1 in the cell membrane were observed between $\mathrm{LNCaP}$ and $\mathrm{C} 4-2 \mathrm{~B}$ cells, indicating that reorganization of asialoGM1 correlates with the invasive phenotype. In addition, further microscopy studies revealed that asialoGM1 and integrin $\alpha 2 \beta 1$ colocalized in $\mathrm{C} 4-2 \mathrm{~B}$ cells. These results suggest a close connection of asialoGM1 with integrin $\alpha 2 \beta 1$, and co-immunoprecipitation of asialoGM1 in C4-2B cells, confirmed the association with integrin $\alpha 2 \beta 1$, while this could not be detected in the parental LNCaP cells.

These observations led us to explore the possibility that asialoGM1 and integrin $\alpha 2 \beta 1$ receptors act in synergy in C4-2B cells to mediate alterations in adhesive, migratory and invasive behavior. While no significant reduction was found in the different biological assays under the conditions used in this study, the idea that asialoGM1 and integrin $\alpha 2 \beta 1$ work together to achieve the phenotypic changes is plausible since signaling molecules found in the co-immunoprecipitates of asialoGM1 were previously identified as being involved in a major signaling pathway leading to expression of MMPs in C4-2B cells (data not shown).

These observations are in line with several studies demonstrating that glycosphingolipids play crucial roles in integrinmediated cellular activities thereby affecting signal transduction pathways and that asialoGM1 is able to engage in cytoplasmic signaling networks $(1,20)$. Moreover, there is a high possibility that GSLs are complexed with tetraspanins at the cell surface. Along this line, tetraspanins CD9 and CD82 are identified as motility inhibitors and decreased expression is reported to be associated with increased invasion and metastasis in prostate cancer $(21,22)$. Although the mechanism largely remains unclear, detailed study on CD9 and CD82 indicate that these tetraspanins alone do not inhibit tumor cell motility or invasiveness rather such inhibition occurs through regulating the biological activities of associated proteins, as integrins, and/or reorganizing the components of the cell membrane
$(1,23)$. Correspondingly, we could not detect CD9 and CD82 in the co-immunoprecipitates of asialoGM1, although both tetraspanins are present on $\mathrm{LNCaP}$ and $\mathrm{C} 4-2 \mathrm{~B}$ cells (data not shown).

In our previous study, we pointed out that the increased cell adhesion and invasion as well as the activation of the downstream signaling pathway is attributed to major alterations in the organizational status of the $\alpha 2 \beta 1$ integrin receptor, rather than to increased expression, and reminded that the observations should not be considered as the mechanism of a single player, but as key components in complex with other crucial molecules (8). Herein, we report that asialoGM1 is reorganized and colocalized with integrin $\alpha 2 \beta 1$ in $C 4-2 B$ cells, suggesting that the interaction of integrin $\alpha 2 \beta 1$ and asialoGM1 is necessary to maintain the invasive phenotype. The increased cell surface expression of asialoGM1 in C4-2B cells as compared to the parental LNCaP cells, however, remains at present unexplained. It has been generally accepted that in most cases the major pool of cellular GSLs is localized in the cell surface membrane. Minor sites of location are the subcellular organelles, where GSL metabolism occurs, or the vesicles, or other transport structures, involved in GSL intracellular traffic (24). Based on the latter, we presume the subcellular localization and/or accumulation of asialoGM1 in $\mathrm{LNCaP}$ cells, this may point out to the existence of specific sorting mechanisms that regulate the intracellular transport and localization of GSLs or the presence of agents that affect the sorting mechanisms between endosomes/Golgi apparatus and the cell membrane and is currently under investigation.

It is not yet clear why particularly asialoGM1 is involved in metastatic potential and not the related GM1, one possible explanation could be the presence of sialidases at the cell membrane that remove sialic acid from sialylated gangliosides (25). In fact, studies indicate that metastatic potential does not always parallel the sialic acid levels, instead there is evidence to believe that the altered sialidase expression is more important for metastases. In addition, increase in neuraminidase NEU3, is often detected in prostate cancer and correlates with the histological differentiation grade (26). Another possibility could be the metabolic recycling of sugar residues of glycosphingolipids, especially of sialic acids, since this is a way of saving energy and channeling sugars to sites of activation, through cytoskeleton microfilaments (24). Further studies based on these possibilities are in progress.

In conclusion, the present study reports that cell surface expression of asialoGM1 correlates with cancer progression and suggests that the reorganization and interaction of asialoGM1 with integrin $\alpha 2 \beta 1$ receptors are crucial in the regulation of cancer metastatic potential. To the best of our knowledge this is the first study to report that asialoGM1 is expressed on prostate cancer cells and that asialoGM1 plays a significant role in prostate cancer progression.

\section{Acknowledgements}

We thank Drs L. Oomen and L. Brocks (Department of Tumor Biology, The Netherlands Cancer Institute, The Netherlands) for assistance with the confocal microscopy analysis. This work was supported by the US National Institutes of Health [RR-16480] under the INBRE program of the National 
Center for Research Resources, the New Mexico Tech startup funds and the New Mexico Department of Veteran Services.

\section{References}

1. Hakomori SI: Structure and function of glycosphingolipids and sphingolipids: recollections and future trends. Biochim Biophys Acta 1780: 325-346, 2008.

2. Hakomori S: Tumor malignancy defined by aberrant glycosylation and sphingo(glycol)lipid metabolism. Cancer Res 56: 5309-5318, 1996.

3. Ruggieri S, Mugnai G, Mannini A, Calorini L, Fallani A, Barletta E, Mannori G and Cecconi O: Lipid characteristics in metastatic cells. Clin Exp Metastases 17: 271-276, 1999.

4. Furukawa K, Hamamura K, Nakashima H and Furukawa K: Molecules in the signaling pathway activated by gangliosides can be targets of therapeutics for malignant melanomas. Proteomics 8: 3312-3316, 2008.

5. Von Holst H, Nygren C, Boström K, Collins VP and Fredman P: The presence of foetal ganglioside antigens 3'-isoLLM1 and 3'6'-isoLD1 in both glioma tissue and surrounding areas of the human brain. Acta Neurochir 139: 141-145, 1997.

6. Hoon DS, Okun E, Neuwirth H, Morton DL and Irie RF: Aberrant expression of gangliosides in human renal cell carcinomas. J Urol 150: 2013-2018, 1993.

7. Ohkawa Y, Miyazaki S, Miyata M, Hamamura K, Furukawa K and Furukawa K: Essential roles of integrin-mediated signaling for the enhancement of malignant properties of melanomas based on the expression of GD3. Biochem Biophys Res Commun 373: 14-19, 2008.

8. Van Slambrouck S, Jenkins AR, Romero AE and Steelant WFA: Reorganization of the integrin $\alpha 2$ subunit controls cell adhesion and cancer cell invasion in prostate cancer. Int J Oncol 34: 1717-1726, 2009.

9. Toledo MS, Suzuki E, Handa K and Hakomori S: Effect of ganglioside and tetraspanins in microdomains on interaction of integrins with fibroblast growth factor receptor. J Biol Chem 280: 16227-16234, 2005.

10. Todeschini AR, Dos Santos JN, Handa K and Hakomori S: Ganglioside GM2-tetraspanin CD82 complex inhibits met and its cross-talk integrins providing a basis for control of cell motility through glycosynapse. J Biol Chem 282: 8123-8133, 2007.

11. Thalmann GN, Sikes RA, Wu TT, Degeorges A, Chang SM, Ozen M, Pathak S and Chung LW: LNCaP progression model of human prostate cancer: androgen-independence and osseous metastasis. Prostate 44: 91-103, 2000.

12. Sigstedt SC, Hooten CJ, Callewaert MC, Jenkins AR, Romero AE, Pullin MJ, Kornienko A, Lowrey TK, van Slambrouck S and Steelant WF: Evaluation of aqueous extracts of Taraxacum officinale on growth and invasion of breast and prostate cancer cells. Int J Oncol 32: 1085-1090, 2008.
13. Geho DH, Bandle RW, Clair T and Liotta LA: Physiological mechanisms of tumor-cell invasion and migration. Physiology 20: 194-200, 2005.

14. Moore ML, Chi MH, Goleniewska K, Durbin JE and Peebles RS Jr: Differential regulation of GM1 and asialo-GM1 expression by $\mathrm{T}$ cells and natural killer (NK) cells in respiratory syncytial virus infection. Viral Immunol 21: 327-339, 2008.

15. Dasgupta S, van Halbeek H and Hogan EL: Ganglio-N-tetraosylceramide (GA1) of bovine and human brain. Molecular characterization and presence in myelin. FEBS Lett 301: 141-144, 1992.

16. Saiman L and Prince A: Pseudomonas aeruginosa pili bind to AsialoGM1 which is increased on the surface of cystic fibrosis epithelial cells. J Clin Invest 92: 1875-1880, 1993.

17. Krivan HC, Ginsburg V and Roberts DD: Pseudomonas aeruginosa and Pseudomonas cepacia isolated from cystic fibrosis patients bind specifically to gangliotetraosylceramide (AsialoGM1) and gangliotriaosylceramide (AsialoGM2). Arc Biochem Biophys 260: 493-496, 1988.

18. De Bentzmann S, Roger P, Dupuit F, Bajolet-Laudinat O, Fuchey C, Plotkowski MC and Puchelle E: Asialo GM1 is a receptor for Pseudomonas aeruginosa adherence to regenerating respiratory epithelial cells. Infect Immun 64: 1582-1588, 1996.

19. Enzan H, Hiroi M, Saibara T, Onishi S, Yamamoto Y, Yamamoto $\mathrm{H}$ and Hara $\mathrm{H}$ : Immunoelectron microscopic identification of asialoGM1-positive cells in adult rat liver. Virchows Arc B Cell Pathol 60: 389-398, 1991.

20. McNamara N and Basbaum C: Signaling networks controlling mucin production in response to Gram-positive and Gramnegative bacteria. Glycoconj J 18: 715-722, 2001

21. Wang JC, Bégin LR, Bérubé NG, Chevalier S, Aprikian AG, Gourdeau $\mathrm{H}$ and Chevrette M: Down-regulation of CD9 expression during prostate carcinoma progression is associated with CD9 mRNA modifications. Clin Cancer Res 13: 2354-2361, 2007.

22. Dong JT, Lamb PW, Rinker-Schaeffer CW, Vukanovic J, Ichikawa T, Isaacs JT and Barrett JC: KAI1, a metastasis suppressor gene for prostate cancer on human chromosome 11p11.2. Science 268: 884-886, 1995.

23. Lazo PA: Functional implications of tetraspanin proteins in cancer biology. Cancer Sci 98: 1666-1677, 2007.

24. Tettamanti G: Ganglioside/glycosphingolipid turnover: new concepts. Glycoconj J 20: 301-317, 2004.

25. Miyagi T, Wada T, Yamaguchi K and Hata K: Sialidase and malignancy: a minireview. Glycoconj J 20: 189-198, 2004.

26. Miyagi T: Aberrant expression of sialidase and cancer progression. Proc Jpn Acad Ser B Phys Biol Sci 84: 407-418, 2008. 